\title{
ISOLAMENTO DE FUNGOS EM ALGUNS PRODUTOS DERIVADOS DE MILHO
}

\section{FUNGI ISOLATED IN SOME CORN DERIVED PRODUCTS}

\author{
Elaine Pittner ${ }^{1}$, Tiago Czervinski ${ }^{2}$, Hermes Francisco Sanches ${ }^{2}$, \\ Marta Chagas Monteiro ${ }^{2 *}$ \\ 1 Universidade Estadual de Maringá - UEM, Programa de Pós-graduação em Análises \\ Clínicas, Maringá, PR \\ 2* Autor para contato: Universidade Estadual do Centro-Oeste - UNICENTRO, Departamento \\ de Farmácia, Guarapuava, PR; (42) 3629-1444, ramal 8137; \\ e-mail:martachagas2@yahoo.com.br.
}

Recebido para publicação em 02/03/2006

Aceito para publicação em 21/03/2007

\section{RESUMO}

O objetivo deste trabalho foi identificar os gêneros de fungos e sua freqüência em três produtos derivados de milho: fubá, quirera e farinha de milho, comercializados em diferentes estabelecimentos do município de Guarapuava, Paraná, Brasil. A maioria das amostras de derivados de milho apresentou contaminação significativa por Penicillium, Aspergillus e Fusarium. As amostras de fubá apresentaram maiores índices ( $>25 \mathrm{ufc} / \mathrm{g}$ ) de contaminação fúngica, provavelmente originária da matéria-prima, condições de processamento e/ou armazenamento do mesmo. Os fungos isolados e identificados nas amostras de fubá, quirera e farinha estão normalmente presentes em produtos e subprodutos armazenados, e alguns deles têm toxigênicos potenciais.

Palavra-chave: Zea mays, produtos derivados de milho, fungos.

\begin{abstract}
The objective of this work was to identify the fungi genera and frequency in three corn derived products: corn meal, cracked corn and corn flour, commercialized in distinct establishments of Guarapuava City, Paraná, Brazil. The samples majority of corn derived products presented significant contamination by Penicillium, Aspergillus and Fusarium. The corn meal presented highest percentage $(>25$ ufc/g) of fungi contamination, probably coming from the corn kernels and/or corn processing and storage conditions. The fungi isolated and identified in samples of
\end{abstract}


corn meal, cracked corn and corn flour were normally found in storage products.

Some of these species have toxigenic potential.

Key words: Zea mays, corn derived products, fungi.

\section{Introdução}

O milho (Zea mays) é uma das culturas de maior importância no Brasil, não só pela extensão da área cultivada, mas por sua diversidade de utilização, em que se destaca a alimentação animal e a humana e por seus reflexos sócio-econômicos. Existem mais de 600 derivados do milho, dos quais, aproximadamente 500 se destinam à alimentação humana (Pinazza, 1993). Os produtos derivados de milho são bastante apreciados na culinária brasileira, tendo participação efetiva como componentes básicos na dieta alimentar das camadas mais pobres da população (Melo-Filho e Richetti, 1997; Lazzari, 1997). Todos os produtos a base de milho são suscetíveis à contaminação por bolores e leveduras. Muitos bolores são potencialmente micotoxigênicos, produzindo toxinas que são prejudiciais à saúde humana (Christensen e Meronuck et al., 1986; Christensen e Meronuck et al., 1989; Champ et al., 1991; Sauer et al., 1992; Abarca et al., 1994; Asevedo et al., 1994; Almeida et al., 2000; Alhadas et al., 2004).

São vários os fatores que afetam o crescimento de fungos em grãos de milho, tais como, teor de umidade dos grãos, condição física e sanitária dos grãos, nível de inoculação do fungo, conteúdo de oxigênio e armazenamento anterior, insetos e ácaros. Os insetos podem iniciar ou agravar o desenvolvimento de fungos, pois pela sua atividade metabólica, ocorre um aumento do teor de umidade e temperatura da massa dos grãos (Banwart, 1981; Christensen e Meronuck, 1986; Watson e Ramstad, 1987; Christensen e Meronuck, 1989; Sinha e Sinha, 1991; Hagstrum e Flinn, 1992; Blank Et Al., 1995; Miller, 1995; Farias et al., 2000).

Quando ocorre a disseminação de fungos nos grãos de milho, ainda no campo ou no armazenamento, os seus derivados, tais como, farinha, fubá e quirera, entre outros, carregarão os esporos e/ou os metabólitos fúngicos, como as micotoxinas (Gelli et al., 1990; Asevedo et al., 1994; Almeida et al., 2000). A contaminação de derivados de milho por fungos representa um problema de saúde pública, uma vez que muitos fungos são potencialmente micotoxigênicos (Asevedo et al., 1994; Almeida et al., 2000; Farias et al., 2000). Os fungos que colonizam grãos e sementes podem ser divididos em dois grupos: i) fungos do campo, que infectam o produto ainda no campo, cujos principais gêneros são: Cephalosporium, Fusarium, Gibberella, Nigrospora, Helminthosporium, Alternaria e Cladosporium que colonizam grãos e sementes durante o amadurecimento e o dano é causado antes da colheita (Watson e Ramstad, 1987; Sinha e Sinha, 1991; Miller, 1995); ii) fungos de armazenamento, que colonizam o milho pouco antes e durante o armazenamento, normalmente são encontrados em grande número em armazéns, moinhos, silos, elevadores, equipamentos e lugares onde são armazenados, manuseados e processados produtos agrícolas. Os principais fungos são: Aspergillus, Penicillium, Rhizopus e Mucor. Os fungos do gênero Aspergillus [A. halophilicus, A. restrictus, A. glaucus, A. candidus, A. alutaceus (A. ochraceus) e A.flavus] e os do gênero Penicillium ( $P$. viridicatum e $P$. verrucosum) são os indicadores de deterioração em sementes e grãos causando danos ao gérmen, descoloração, alterações nutricionais, perda da matéria seca e os primeiros estágios da deterioração microbiológica (Banwart, 1981; Watson e Ramstad, 1987; Sinha e Sinha, 1991).

O objetivo deste trabalho foi identificar os gêneros de fungos e sua freqüência em amostras de derivados de milho, como o fubá, farinha e quirera, de três fabricantes diferentes, comercializados em dois estabelecimentos do município de Guarapuava-PR.

\section{Materiais e métodos}

A avaliação micológica foi qualitativa e quantitativa, sendo realizada em três alimentos 
derivados de milho: fubá, farinha e quirera. Neste trabalho, utilizaram-se três amostras de cada derivado, sendo cada uma de um fabricante diferente, totalizando três por derivado, que foram adquiridas de dois estabelecimentos diferentes. Utilizou-se nos experimentos $1.000 \mathrm{~g}$ por amostras de cada fabricante, sendo cada uma de $3000 \mathrm{~g}$, originalmente. As amostras foram homogeneizados, sendo destas retiradas $10 \mathrm{~g}$ para a realização da diluição seriada (1:10 e 1:100). De cada uma das diluições, semeou-se alíquotas de $0,1 \mathrm{~mL}$ por placa, cultivando-as por intermédio de duas metodologias: a) em superfície do meio de cultura, com auxilio da alça de Drigalski e b) em profundidade, na qual foi inoculada com auxílio de uma micropipeta, $0,1 \mathrm{~mL}$ das diluições, no fundo da placa e posteriormente colocado o meio de cultura sobre a amostra. Ambas metodologias foram realizadas em duplicata em placas contendo aproximadamente $20 \mathrm{~mL}$ de meio Agar Batata Dextrose (BDA) (Ribeiro et al., 2003). As placas permaneceram em temperatura ambiente $\left(28^{\circ} \mathrm{C} \pm 1{ }^{\circ} \mathrm{C}\right)$ por sete dias (Abarca et al., 1994). A identificação dos gêneros dos fungos foi realizada através de características macroscópicas e microscópicas (Barnett, 1962; Silveira, 1981; Lacaz et al., 1994; Larone, 1995), sendo que simultaneamente foi realizada a contagem padrão em placas e, posteriormente, feita à média dos resultados obtidos. Os resultados apresentados nas figuras foram expressos em unidade formadora de colônia por grama (UFC/g).

\section{Resultados e discussão}

Em todas as amostras dos derivados de milho (fubá, farinha de milho e quirera) foram encontrados fungos filamentosos, pelas duas metodologias: profundidade e superfície. Todas as amostras de farinha de milho e quirera apresentaram um valor inferior a 5 x 10 $\mathrm{UFC} / \mathrm{g}$ de fungos tanto pela metodologia de superfície quanto pela de profundidade (Figuras $1 \mathrm{e} 2$ ). Entretanto, as amostras de fubá não foram tão homogêneas quanto os demais derivados, uma vez que das três amostras avaliadas, a terceira amostra apresentou aumento significativo no número de $\mathrm{UFC} / \mathrm{g}$, quando comparada às demais amostras (amostra 1 e 2: UFC/g $<5$ x 10; amos- tra 3: UFC/g $>25 \times 10$ por metodologia de profundidade; amostra 1 e 2: UFC/g < 10 x 10; amostra 3: $\mathrm{UFC} / \mathrm{g}>25 \times 10$ pela metodologia de superfície). Estes dados mostram que todas as amostras de fubá encontravam-se com contaminação fúngica acima dos padrões preconizados pela legislação brasileira [conforme Resolução da ANVISA (Agência Nacional de Vigilância Sanitária) - CNNPA(Comissão Nacional de Normas e Padrões para Alimentos) n ${ }^{\circ} 12$ de 1978, D.O. de 24/07/1978].

Semeados em Profundidade

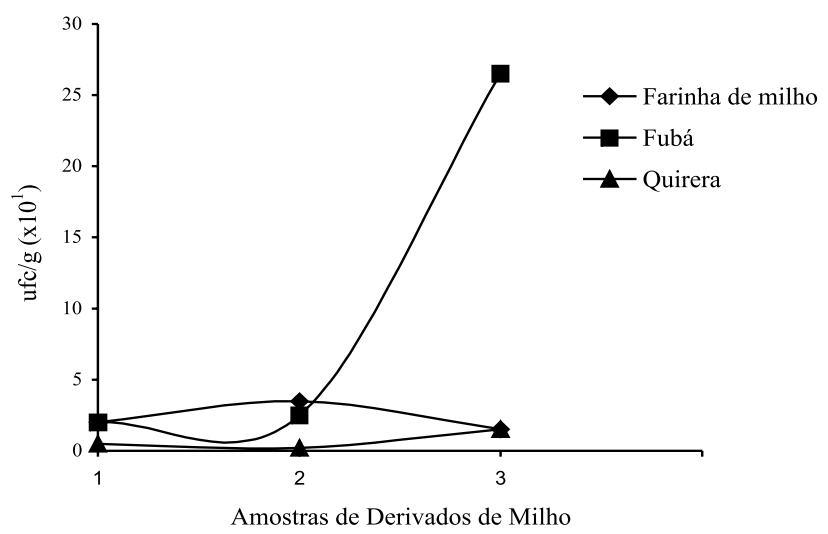

Figura 1 - Número médio de ufc/g de fungos em três amostras de alimentos derivados de milho (farinha, fubá e quirera) semeadas em profundidade em meio BDA.

Semeados em Superfície

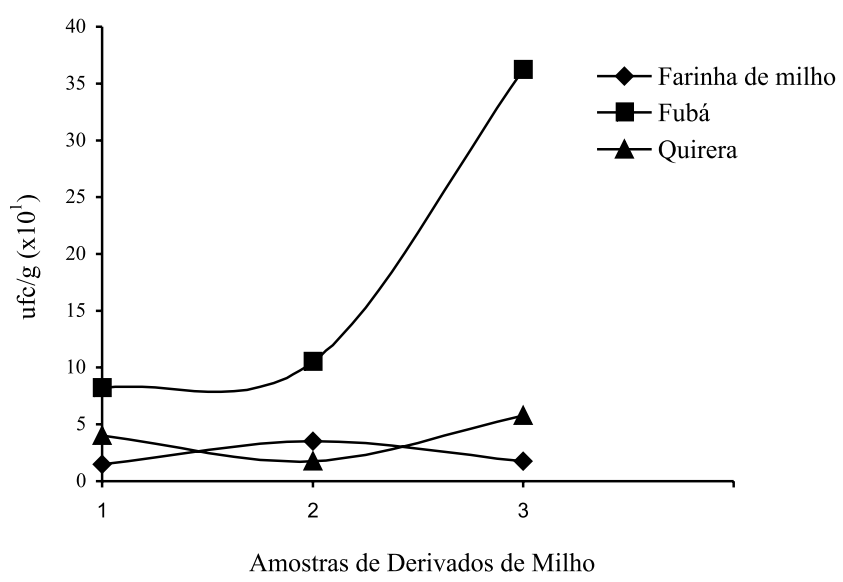

Figura 2 - Número médio de ufc/g de fungos em três amostras de alimentos derivados de milho (farinha, fubá e quirera) semeadas em superfície em meio BDA. 
Os resultados foram similares aos relatados por Alhadas et al. (2004), que analisaram cinco marcas de fubá comercializadas em Curitiba, Paraná, e verificaram que todas apresentavam contaminação por bolores e leveduras. Estes autores observaram a presença de sete gêneros distintos de bolores: Aspergillus, Penicillium, Cladosporium, Rhizopus, Acremonium, Paecilomyces e Cunninghamela. Destes, os gêneros Aspergillus e Penicillium são potencialmente micotoxigênicos.

Observou-se que o fungo filamentoso mais freqüente, na farinha de milho, foi o gênero Penicillium ( $100 \%$ das amostras), tanto pelo método de profundidade quanto o de superfície. O gênero Fusarium apresentou um índice percentual de $70 \%$ nas amostras por ambos os métodos. Entretanto, o gênero Aspergillus foi encontrado em $70 \%$ das amostras pelo método de profundidade e somente em $30 \%$ pelo método de superfície. Já o gênero Rhizopus não foi encontrado pelo método de profundidade, mas apareceu em 30\% das amostras pelo método de superfície em placa (Figuras 3 e 4, respectivamente).

Com relação à quirera, observou-se que a freqüência encontrada de Penicillium sp foi de $70 \%$ das amostras por método de profundidade e $100 \%$ por método de superfície. O Fusarium sp foi encontrado em $30 \%$ por profundidade e $100 \%$ por superfície. O Aspergillus sp foi encontrado em $70 \%$ das amostras em ambas as metodologias. No entanto, o Rhizopus sp não foi encontrado nas amostras avaliadas (Figuras 3 e 4 , respectivamente).

Encontrou-se em 100\% das amostras de fubá, os gêneros Penicillium sp e Fusarium sp por ambas as metodologias. O gênero Aspergillus sp somente foi encontrado em $30 \%$ das amostras pelo método de profundidade, mas em $100 \%$ das amostras pelo método de superfície. Já o Rhizopus sp foi encontrado em 30\% das amostras por ambas as metodologias (Figuras $3 \mathrm{e}$ 4, respectivamente).

\section{Semeado em Profundidade}

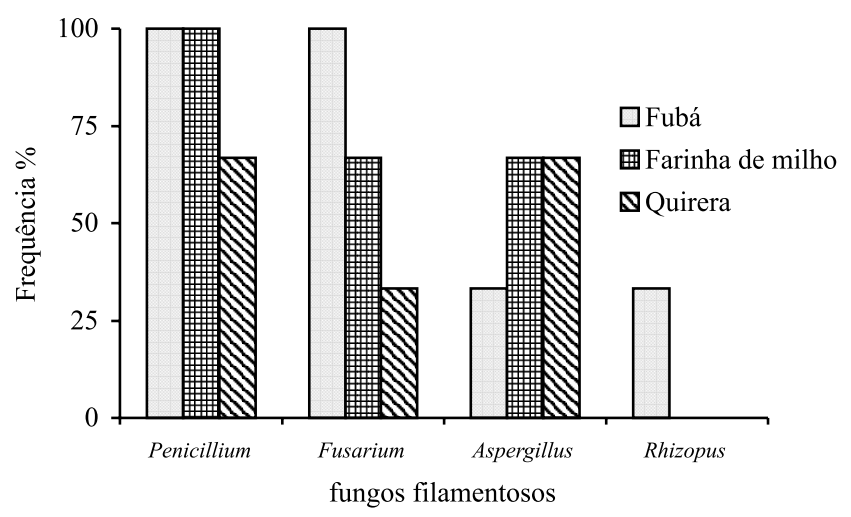

Figura 3 - Freqüência de quatro gêneros de fungos filamentosos (Penicillium, Fusarium, Aspergillus e Rhizopus) em derivados de milho (fubá, farinha e quirera) semeados em profundidade em meio BDA.

\section{Semeado em Superfície}

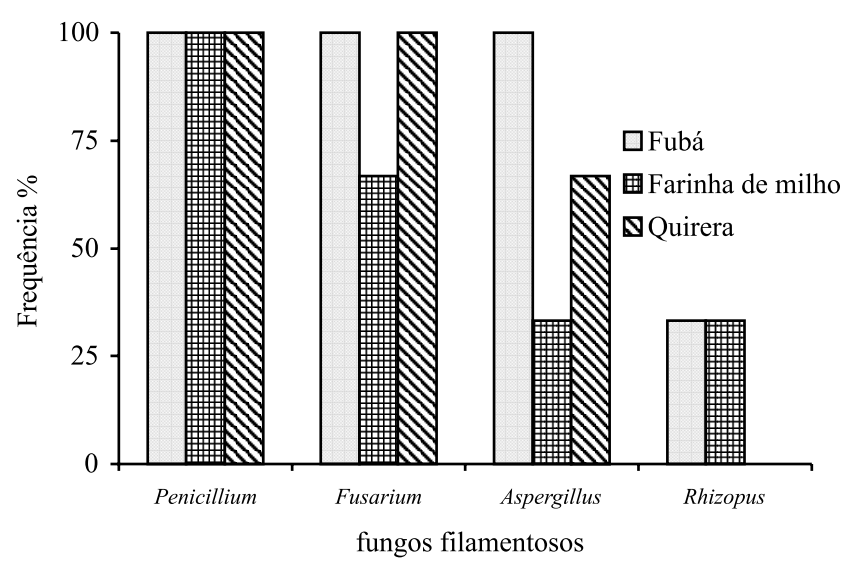

Figura 4 - Freqüência de quatro gêneros de fungos filamentosos (Penicillium, Fusarium, Aspergillus e Rhizopus) em derivados de milho (fubá, farinha e quirera) semeados por método de superfície em meio BDA.

Dentre os gêneros observados, o Fusarium é considerado um fungo de campo, que invade grãos e sementes durante o amadurecimento, sendo o dano 
causado antes da colheita. Este fungo não se desenvolve durante o armazenamento, exceto ocasionalmente, em milho armazenado com alto teor de umidade ou que foi re-umidificado (Marcia e Lazzari,1998). O gênero Aspergillus [A. halophilicus, A.restrictus, A. glaucus, $A$. candidus, A. alutaceus (A. ochraceus) e $A$. flavus] e Penicillium (P. viridicatum e $P$. verrucosum) são indicadores de deterioração em sementes e grãos causando danos ao gérmen, descoloração, alterações nutricionais, perda da matéria seca e os primeiros estágios da deterioração microbiológica (Sinha e Sinhá, 1991; Samsom, 1991; Sinha e Sinha, 1992; Miller, 1995).

Marcia e Lazzari (1998), descreveram que as amostras de fubá avaliadas em seu estudo, apresentaram elevada contaminação fúngica. Sendo que, predominaram: Penicillium, Aspergillus, Fusarium, Mucore Rhizopus em ordem decrescente de importância. Acredita-se, que a grande incidência de contaminação por fungos em fuba, seja decorrente das condições ambientais (manuseio, processamento e armazenamento) e qualidade da matéria prima.

Ribeiro et al. (2003) encontraram 16 espécies, representantes de oito gêneros, em amostras de fubá, sendo o maior número de espécies isoladas, até então, entre os produtos analisados. Salienta-se, ainda, que o Penicillium foi o gênero predominante nestas amostras, para o qual foi diagnosticado um total de oito espécies. Além deste grupo, a espécie Fusarium moniliforme foi encontrada nas amostras tanto pelo método de profundidade quanto de superfície, nos três níveis de diluições empregados. Na farinha de milho, foram isoladas oito espécies distribuídas em quatro gêneros, sendo o menor número de espécies entre os produtos analisados. Quanto à quirera, constataramse 10 espécies isoladas, representadas por três gêneros, sendo que a maioria das espécies pertencia ao gênero Penicillium. Os gêneros Penicillium e Rhizopus foram encontrados em grande parte dos produtos analisados, destacando-se o Penicillium como dominante em todos os produtos do milho. Ribeiro et al. (2003) também evidenciaram a predominância das espécies Penicillium funiculosum e $P$. Duclauxii, seguidas das espécies $P$. brevicompactum e P. Aurantiogriseum.

Visto que a contaminação dos alimentos por fungos toxigênicos e produção de micotoxinas, geralmente está relacionada às condições ambientais de cultivo e armazenamento, investigações contínuas de sua ocorrência se tornam necessárias (Norrede, 1992; Bullerman e Tsai, 1994). Os trabalhos de ocorrência de fungos e micotoxinas em alimentos consumidos no Brasil, têm contribuído para avaliação e estudos de medidas a serem tomadas, a fim de prevenir a contaminação. Já foram relatadas altas incidências de toxinas em alimentos, tais como: i) amendoim, detectou-se aflatoxinas; ii) produtos de arroz, trigo e derivados de milho, apresentaram aflatoxinas, zearalenona, ocratoxina e zearalenona, desoxinivalenol e toxina T-2 e iv) em farinhas, tricotecenos (Purwoko et al., 1991; Bottalico et al., 1995; Oliveria et al., 2002).

Almeida et al. (2000) estudaram a microbiota fúngica de 66 amostras de três híbridos de grãos de milho recém-colhido, provenientes de três regiões do Estado de São Paulo - Brasil (Assis, Capão Bonito e Ribeirão Preto) e verificaram a predominância de Fusarium spp, Penicillium spp e Aspergillus spp e outros dois gêneros de fungos filamentosos, isolados de grãos com teor de umidade entre $5 \%$ e $18 \%$. Do gênero Fusarium spp, a espécie $F$. moniliforme foi a mais freqüentemente isolada, enquanto que, em relação ao gênero Aspergillus, predominou A. flavus nas três regiões. Todas as cepas de $F$. moniliforme isoladas (40), produziram fumonisinas, que variaram de $20 \mathrm{a}$ $2168 \mathrm{mg} / \mathrm{g}\left(\mathrm{FB}_{1}\right)$ e 10 a $380 \mathrm{mg} / \mathrm{g}\left(\mathrm{FB}_{2}\right)$. Das 10 cepas de Aspergillus flavus isoladas, seis destas (60,0\%) produziram aflatoxinas, que variaram de 615 a 30.750 $\mathrm{mg} / \mathrm{kg}\left(\mathrm{AFB}_{1}\right)$ e 11 a $22 \mathrm{mg} / \mathrm{kg}\left(\mathrm{AFB}_{2}\right)$.

Pode-se observar que vários gêneros de fungos com elevado poder toxigênicos foram encontrados em alimentos derivados de milho, tais como fubá, farinha de milho e quirera.

\section{Conclusão}

Os gêneros de fungos filamentosos encontrados nos derivados de milho foram: Penicillium sp, Rhizopus sp, Fusarium sp e Aspergillus sp. O fubá foi o alimento que apresentou o maior índice de UFC/g, tanto pelo método de profundidade quanto de superfície. A 
disseminação de fungos em alimentos pode ocorrer provavelmente devido às condições ambientais favoráveis ao crescimento.

\section{REFERÊNCIAS}

1. ABARCA, M.L.; BRAGULAT, M.R.; CASTELLA, G.; CABANES, F.J. Mycoflora and aflatoxin-producing strains in animal mixed feeds. J. Food Prot., vol. 57, n. 3, 1994. p.256-258.

2. ALMEIDA, A.P; CORREA, B; MALLOZZI, M.A.B. Mycoflora and aflatoxin/fumonisin production by fungal isolates from freshly harvested corn hybrids. Braz. J. microbiol., vol. 31, n. 4, 2000, p.321-326.

3.ALHADAS, R.V; STUART, R.M.; BEUX, M.R; PIMENTEL, I.C. Contagem de Bolores e leveduras em fubá e identificação de gêneros potencialmente toxigênicos. Vis. Acad., Curitiba, vol. 5, n. 2, 2004, p.79-82.

4.ASEVEDO, I.G.; GAMBALE, W.; CORRÊA, B.; PAULA, C.R.; ALMEIDA, R.M.A.; SOUZA, V.M. - Mycoflora and aflatoxigenic species of Aspergillus ssp isolated from stored maize. Rev. Microbiol., vol. 25, n. 1, 1994, p.46-50.

5. BANWART, G.J. Basic food microbiology. Westport, CT: The AVI Publis.Comp., Inc., 1981, p.51-62.

6. BARNETT, H. L. Illustr. gen. of imper. fungi. 2nd ed.Burgess, 225p, 1962.

7. BLANK, G.; GOSWAMI, N.; MADRID, F.; MARQUARDT, R.R.; FROHLICH, A.A. Evaluation of Tribolium castaneum (Herbst) (Coleoptera: Tenebrionidae) excreta on ochratoxin production in stored wheat. J. Stored Prod. Res., vol. 31, n.2,1995, p.151-155.

8. BOTTALICO, A.; LOGRIECO, A.; RITIENI, A.; MORETTI, A.; RANDAZZO, G.; CORDA, P. Beauvericin and fumonisin $\mathrm{B}_{1}$ in preharvest Fusarium moniliforme maize ear rot in Sardinia. Food Addit. Contam., vol. 12, n. 4, 1995, p.599-607.

9. BULLERMAN, L.B.; TSAI, W-Y.J. Incidence and levels of Fusarium moniliforme, Fusarium proliferatum and fumonisins in corn and corn-based foods and feeds. J. Food Prot., vol. 57, n. 6, 1994, p.541-546.

10. CHAMP, B.R.; HIGHLEY, E.; HOCKING,A.D.; PITT, J.I. ed. Fungi and mycotoxins in stored products. In: Inter. Conf., Bangkok, Thailand, ACIAR Proceedings, n. 36, 1991, p270.

11. CHRISTENSEN, C.M.; MERONUCK, R.A. Quality Maintenance in Stored Grains and Seeds. Univer. MN Press, 1986, p.150.

12. CHRISTENSEN, C.M.; MERONUCK, R.A. Dry matter loss in yellow dent corn resulting from invasion by storage fungi. Plant Dis., vol. 73, n. 6, 1989, p.501-503.

13. FARIAS, A. X.; ROBBS, C. F.; BITTENCOURT, A. M.;
ANDERSEN, P. M.; CORRÊA, T.B.S. Contaminação endógena por Aspergillus spp. Em milho pós-colheita no Estado do Paraná. Pesq. Agrop. Bras., vol.35, n.3, 2000, p.617-621.

14. GELLI, D.S.; JAKABI, M.; PORTO, E. Isolamento de Aspergillus spp aflatoxigênicos de produtos alimentícios - São Paulo, Capital. Rev. Inst. Adolfo Lutz, vol. 50, n.1, 1990, p.319323.

15. HAGSTRUM, D.W.; FLINN, P.W. Integrated pest management of stored-grain insects. In: SAUER, D.B. (ed.). Storage of cereal grains and their products. St. Paul, MN, Amer. Assoc. of Cereal Chem., 1992, p607.

16. LACAZ, C.S.; PORTO; E.; MARTINS; J.E.C. Microb. Médica: fungos, actinomicetos e algas de interesse médico. São Paulo, Editora Sarvier, 1991.

17. LARONE, D.H. Medic. import. fungi: a guide to ident., 3.ed. Washington, P. C. ASM Press, Harper \& Row, 1995.

18. LAZZARI, F.A. Umidade, fungos e micotoxinas na qualidade de sementes, grãos e rações. $2^{a}$ Edição. Curitiba, Ed. do Autor, 1997, p140.

19. MARCIA, B.A.; LÁZZARI, F.A. Monitoramento de fungos em milho em grão, grits e fubá, Ciênc. Tecnol. Aliment. vol 18, n.4, 1998.

20. MELO-FILHO, G.A.; RICHETTI. A. Aspectos socioeconômicos da cultura de milho. Embrapa, Circ. Téc., vol.5, 1997, p.12-21.

21. MILLER, J.D. Fungi and mycotoxins in grain: implications for stored product research. J. Stored Prod. Res., vol. 31, n. 1, 1995, p.1-16.

22. NORRED, W.P. - Fumonisins - Mycotoxins produced by Fusarium moniliforme . J. Toxicol. Environ. Health, vol. 38,1992, p.309-328.

23. OLIVEIRA, M.S. De; PRADO, G.; ABRANTES, F.M.; SANTOS, L.G.; RIALA, T.V. Incidence of aflatoxins, Deoxynivalenol and Zearalenone in products commercialized in the cites of Minas Gerais state in 1998-2000, Rev. Inst. Adolfo Lutz, vol. 61, n.1, 2002, p. 1-6.

24. PEREIRA, P.R.V.S. Comparação entre métodos para detecção de Coleópteros adultos (Insecta: Coleoptera) e ocorrência de fungos em trigo armazenado. Curitiba, 1994. 86p. (Mestrado em Ciências Biológicas), Área de Concentração em Entomologia da Universidade Federal do Paraná - UFPR.

25. PINAZZA, L.A. Perspectiva do milho e do sorgo no Brasil. In Cultura do milho: fatores que afetam a produtividade (L.T. Bull \& H. Cantarella, eds.). Assoc. Brás. para Pesq. da Potassa e do Fosfato, Piracicaba, 1993, p.1-10.

26. PURWOKO, H.M.; HALD, B.; WOLSTRUP, J. Aflatoxin content and number of fungi in poultry feedstuffs from Indonesia. Lett. Appl. Microbiol., vol. 12, 1991, p.212-215. 
27. RIBEIRO, S.A.A.L.; CAVALCANTI, M.A.Q.;FERNANDES, M.J.S. e LIMA, D.M.M. Fungos filamentosos isolados de produtos derivados do milho comercializados em Recife, Pernambuco. Revis. Brasil. Bot., vol. 26, n.2, 2003, p. 223-229.

28. SAMSOM, R.A. Identification of food-borne Penicillium. Aspergillus and Fusarium species. In: CHAMP, B.R.; HISHLEY, E.; HOCKING, A.D.; PITT, J.I. ed. Fungi and mycotoxins in stored products. In: Inter. Conf. Bangkok, Thailand, ACIAR Proceedings, n. 36, 1991, p. 270.

29. SAUER, D.B.; MERONUCK, R.A.; CHRISTENSEN, C.M. Microflora. In: SAUER, D.B. (ed.). Storage of cereal grains and their products. St. Paul, MN, Amer. Assoc. of Cereal Chemists,1992, p. 607.
30. SILVEIRA, V.D. Micologia. Rio de Janeiro, Editora Interamericana, 325p,1981.

31. SINHA, K.K.; SINHA, A.K. Impact of stored garin pests on seed deterioration and aflatoxin contamination in maize. $\mathbf{J}$. Stored Prod. Res., vol. 28, n. 3, 1991, p.211-219.

32. SINHA, K.K.; SINHA, A.K. Effect of Sitophilus oryzae infestation on Aspergillus flavus infection and aflatoxin contamination in stored wheat. J. Stored Prod. Res., vol. 27, n. 1,. 1992, p.65-68.

33. WATSON, S.A.; RAMSTAD, P.E. Chemistry and Technology. St. Paul, MN: Amer. Assoc. of Cereal Chemists, 1987, p.605. 